\title{
THE STRONG LAW OF LARGE NUMBERS FOR MULTIVARIATE FUNCTIONS OF CONTINUOUS-STATE NONHOMOGENEOUS MARKOV CHAINS
}

\author{
PENGYAN ZHANG ${ }^{1}$, WEIGUO YANG ${ }^{2}$, AND BEI WANG ${ }^{3}$ \\ ${ }^{1,2,3}$ Faculty of Science \\ Jiangsu University \\ Zhenjiang 212013, P.R. CHINA
}

\begin{abstract}
In this paper, we are going to study the strong law of large numbers for multivariate functions of continuous-state nonhomogeneous Markov chains. Firstly, we give a primary proof of equivalence of the ergodicities for continuous-state homogeneous Markov chains. Then, we establish some lemmas which are the basis of the main result. Finally, we study the strong law of large numbers for functions of $N+1$ variables of continuous-state nonhomogeneous Markov chains.
\end{abstract}

AMS Subject Classification: 60F15, 60J05

Received: September 26, 2017; Accepted: November 10, 2017 ;

Published: March 11, 2018 doi: 10.12732/dsa.v27i2.4

Dynamic Publishers, Inc., Acad. Publishers, Ltd. https://acadsol.eu/dsa

\section{INTRODUCTION}

For any $x \in R$, let $p(x, y)$ be a nonnegative Borel measurable function, and

$$
\int_{R} p(x, y) d y=1
$$

$p(x, y)$ will be called transition probability density. If $p\left(x_{1}, y\right)=p\left(x_{2}, y\right)$ for any $x_{1}, x_{2} \in R, p(x, y)$ will be called constant transition density. If $p(x, y)$ is a constant transition density, then $p(x, y)=\pi(y)$ is a probability density.

Let $\left\{X_{n}, n \geq 0\right\}$ be a nonhomogeneous Markov chain taking values in $R$ with the transition kernels $P_{n}(x, B)\left(P_{n}(x, B)=\mathbf{P}\left(X_{n+1} \in B \mid X_{n}=x\right)\right.$, where $\left.B \in \mathcal{B}(R)\right)$. 
Let $p_{n}(x, y)$ be the transition probability densities. If

$$
P_{n}(x, B)=\int_{B} p_{n}(x, y) d y
$$

$\left\{X_{n}, n \geq 0\right\}$ will be called a continuous-state nonhomogeneous Markov chain.

Let

$$
p^{(n, n+2)}(x, y)=\int_{R} p_{n}(x, z) p_{n+1}(z, y) d z
$$

and

$$
p^{(n, n+k)}(x, y)=\int_{R} p^{(n, n+k-1)}(x, z) p_{n+k-1}(z, y) d z,
$$

then $p^{(n, n+k)}(x, y)$ is also a transition probability density. It is easy to see that for any $B \in \mathcal{B}(R)$,

$$
\mathbf{P}\left(X_{n+k} \in B \mid X_{n}=x\right)=\int_{B} p^{(n, n+k)}(x, y) d y .
$$

If the continuous-state Markov chain is homogeneous, $p_{n}(x, y)$ will be denoted simply by $p$ and $p^{(n, n+k)}$ by $p^{(k)}$, and we have

$$
p^{(n+k)}(x, y)=\int_{R} p^{(n)}(x, z) p^{(k)}(z, y) d z .
$$

$p^{(n)}(x, y)$ is called $n$-step transition probability density of the homogeneous Markov chains.

Let $f(x)$ be a Borel measurable function defined on $R$, we define the norm $\|\cdot\|$ of $f(x)$ as follows:

$$
\|f(x)\|=\int_{R}|f(x)| d x .
$$

There have been some works on strong law of large numbers for nonhomogeneous Markov chains. Liu and Liu [3] have studied the strong law of large numbers for multivariate functions of countable nonhomogeneous Markov chains. Liu and Liu [4] have studied a class of strong law of large numbers for functionals of countable nonhomogeneous Markov chains. Liu and Yang [6] have studied the strong law of large numbers and Shannon-McMillan-Breiman theorem for finite nonhomogeneous Markov chains. Yang [11] has studied the convergence in the Cesàro sense and strong law of large numbers for bivariate functions of countable nonhomogeneous Markov chains under the condition $\lim _{n \rightarrow \infty}(1 / n) \sum_{k=1}^{n}\left\|P_{k}-P\right\|=0$ where $P$ is periodic strongly ergodic. Yang [12] has also studied strong law of large numbers for countable nonhomogeneous Markov chains under the condition of uniform convergence in the 
Cesàro sense which is different from the result of [11]. Dietz and Sethuraman [1] have studied large deviations for a class of finite nonhomogeneous Markov chains.

In this paper, we are going to study the strong law of large numbers for multivariate functions of continuous-state nonhomogeneous Markov chains. Firstly, we give a primary proof of equivalence of the ergodicities for continuous-state homogeneous Markov chains. Then, we establish some lemmas which are the basis of the main result. Finally, we study the strong law of large numbers for multivariate functions of continuous-state nonhomogeneous Markov chains. As corollaries, we give a strong law of large numbers for functions of two variables of continuous-state nonhomogeneous Markov chains.

This paper is organized as follows: In Section 2, we give a primary proof of equivalence of the ergodicities for continuous-state homogeneous Markov chains. In Section 3, we study some lemmas which are the basis of the main result, then we obtain the strong law of large numbers for multivariate functions of continuous-state nonhomogeneous Markov chains.

\section{A PRIMARY PROOF OF ERGODICITIES FOR CONTINUOUS-STATE HOMOGENEOUS MARKOV CHAINS}

In this section, we introduce the definitions of geometric strongly ergodic, strongly ergodic and weakly ergodic for continuous-state homogeneous Markov chains, then we give a primary proof of equivalence of above three ergodicities for continuous-state homogeneous Markov chains.

Definition 2.1. ([9], Definition 2) Let $p(x, y)$ be the transition probability density. Define Dobrushin coefficient of $p$ as follows:

$$
C(p)=\frac{1}{2} \sup _{x, y} \int_{R}|p(x, z)-p(y, z)| d z .
$$

Definition 2.2. Let $\left\{X_{n}, n \geq 0\right\}$ be a continuous-state homogeneous Markov chain with the transition probability density $p(x, y)$. Let $p^{(n)}(x, y)$ be the $n$-step transition probability density, and let $\pi(y)$ be a probability density on $R$.

(i) If there exist two constants $c>0,0<r<1$ and a positive integer $n_{0}$ such that as $n \geq n_{0}$, for any $x$,

$$
\int_{R}\left|p^{(n)}(x, y)-\pi(y)\right| d y \leq c r^{n},
$$

$\left\{X_{n}, n \geq 0\right\}$ will be called geometric strongly ergodic. We also call that $p(x, y)$ is geometric strongly ergodic with probability density $\pi(y)$. (see [7]). 
(ii) If

$$
\sup _{x} \int_{R}\left|p^{(n)}(x, y)-\pi(y)\right| d y \rightarrow 0(n \rightarrow \infty),
$$

$\left\{X_{n}, n \geq 0\right\}$ will be called strongly ergodic. We also call that $p(x, y)$ is strongly ergodic with probability density $\pi(y)$. (see [9]).

(iii) $\left\{X_{n}, n \geq 0\right\}$ will be called weakly ergodic if $C\left(p^{(n)}\right) \rightarrow 0$ as $n \rightarrow \infty$. (see [9]).

Definition 2.3. Let $\left\{X_{n}, n \geq 0\right\}$ be a continuous-state homogeneous Markov chain with the transition probability density $p(x, y)$. Let $\pi(y)$ be a probability density on $R$. If

$$
\pi(y)=\int_{R} \pi(x) p(x, y) d x
$$

$\pi(y)$ will be called stationary distribution of this Markov chain. We also called $\pi(y)$ is the stationary distribution determined by $p$.

If $\left\{X_{n}, n \geq 0\right\}$ is strongly ergodic with the probability density $\pi(y)$, it is easy to see that $\pi(y)$ is the stationary distribution of $\left\{X_{n}, n \geq 0\right\}$.

In the remark of Definition 5 of [9], the author of this paper pointed out that $C(p)=0$ if and only if $p$ is a constant transition density. It is easy to see that this conclusion is not correct. Here we give a revised lemma as follows.

Lemma 2.1. Let $p(x, y)$ be a transition probability density. $C(p)=0$ if and only if there exists a constant transition density $\bar{p}(y)$ such that

$$
\sup _{x} \int_{R}|p(x, y)-\bar{p}(y)| d y=0 .
$$

Proof. Sufficiency is obvious. We only need to prove necessity. Let $\bar{p}(y)=p(0, y)$, it is easy to see that $\bar{p}(y)$ is a constant transition density. Since $C(p)=0$, we have $\frac{1}{2} \sup _{x_{1}, x_{2}} \int_{R}\left|p\left(x_{1}, y\right)-p\left(x_{2}, y\right)\right| d y=0$, and (2.5) holds.

From above lemma, if $C(p)=0$, we can regard $p$ as a constant transition density or a probability density.

Lemma 2.2. ([9], Lemma 6) Let $p(x, y)$ be a transition probability density and let $r(x)$ be a Borel function such that $\|r\|<\infty$ and $\int_{R} r(x) d x=0$. Let

$$
r p(y)=\int_{R} r(x) p(x, y) d x,
$$

then

$$
\|r p\| \leq\|r\| C(p)
$$


Remark. In Lemma 6 of [9], the author has just given this conclusion but not provided a detailed proof. Here we give a completed proof as follows.

Proof. Since $r(x)=r^{+}(x)-r^{-}(x),|r(x)|=r^{+}(x)+r^{-}(x)$, where $r^{+}(x)=$ $\max \{r(x), 0\}, r^{-}(x)=\max \{-r(x), 0\}$. Let $\int_{R} r(x) d x=0$, we have

$$
\int_{R} r^{+}(x) d x=\int_{R} r^{-}(x) d x, \text { and } \int_{R}|r(x)| d x=2 \int_{R} r^{+}(x) d x .
$$

It is easy to see that $\int_{R}\left(\int_{R} r(x) p(x, y) d x\right) d y=0$. Similarly, we have

$$
C(p)=\sup _{x, y} \int_{R}(p(x, z)-p(y, z))^{+} d z
$$

Let

$$
E=\left\{y: \int_{R} r(x) p(x, y) d x>0\right\} .
$$

Since $\int_{R}|r(x)| d x \int_{E} p(x, y) d y<\infty$, by Fubini theorem, we have

$$
\begin{aligned}
\|r p\| & =\int_{R}\left|\int_{R} r(x) p(x, y) d x\right| d y \\
& =2 \int_{R}\left(\int_{R} r(x) p(x, y) d x\right)^{+} d y \\
& =2 \int_{E}\left(\int_{R} r(x) p(x, y) d x\right) d y \\
& =2 \int_{R} r(x) d x \int_{E} p(x, y) d y \\
& =2 \int_{R}\left(r^{+}(x)-r^{-}(x)\right) d x \int_{E} p(x, y) d y \\
& \leq 2\left(\int_{R} r^{+}(x) d x \sup _{x_{1}} \int_{E} p\left(x_{1}, y\right) d y-\int_{R} r^{-}(x) d x \inf _{x_{2}} \int_{E} p\left(x_{2}, y\right) d y\right) \\
& =2 \int_{R} r^{+}(x) d x \sup _{x_{1}, x_{2}} \int_{E}\left(p\left(x_{1}, y\right)-p\left(x_{2}, y\right)\right) d y \\
& \leq 2 \int_{R} r^{+}(x) d x \sup _{x_{1}, x_{2}} \int_{R}\left(p\left(x_{1}, y\right)-p\left(x_{2}, y\right)\right)^{+} d y \\
& =\|r\| C(p) .
\end{aligned}
$$

The proof of this lemma is completed.

Corollary 2.1. (Dobrushin inequality) Let $p(x, y)$ be a transition probability density of a continuous-state homogeneous Markov chain, and let $\rho_{1}(x)$ and $\rho_{2}(x)$ be two probability densities. Then

$$
\left\|\int_{R} \rho_{1}(x) p(x, *) d x-\int_{R} \rho_{2}(x) p(x, *) d x\right\| \leq C(p)\left\|\rho_{1}-\rho_{2}\right\| .
$$


Proof. Let $r(x)=\rho_{1}(x)-\rho_{2}(x)$ in Lemma 2.2, then $\int_{R}\left(\rho_{1}(x)-\rho_{2}(x)\right) d x=0$. By Lemma 2.2, we have

$$
\begin{aligned}
\left\|\int_{R} \rho_{1}(x) p(x, *) d x-\int_{R} \rho_{2}(x) p(x, *) d x\right\| & =\left\|\left(\rho_{1}-\rho_{2}\right) p\right\| \\
& \leq C(p)\left\|\rho_{1}-\rho_{2}\right\| .
\end{aligned}
$$

That is (2.9) holds.

Lemma 2.3. ([8], Lemma 2) Let $p$ and $q$ be two transition probability densities. Then

$$
C(p q) \leq C(p) C(q)
$$

Lemma 2.4. Let $\left\{X_{n}, n \geq 0\right\}$ be a continuous-state homogeneous Markov chain with the transition probability density $p(x, y)$. Then $\left\{X_{n}, n \geq 0\right\}$ is weakly ergodic if and only if there exists a positive integer number $n_{0}$ such that $C\left(p^{\left(n_{0}\right)}\right)<1$.

Proof. The necessity is obvious. We only need to prove sufficiency. Since $p^{(n+1)}(x, y)=$ $\int_{R} p(x, z) p^{(n)}(z, y) d z$, by $(2.11)$, we have $C\left(p^{(n+1)}\right) \leq C(p) C\left(p^{(n)}\right) \leq C\left(p^{(n)}\right)$. Hence $\left\{C\left(p^{(n)}\right)\right\}$ is a nondecreasing sequence. Let $C\left(p^{\left(n_{0}\right)}\right)=\delta<1$, we have $C\left(p^{\left(k n_{0}\right)}\right) \leq$ $\delta^{k} \rightarrow 0$ as $k \rightarrow \infty$. Since $\left\{C\left(p^{\left(k n_{0}\right)}\right)\right\}$ is a subsequence of $\left\{C\left(p^{(n)}\right)\right\}$, hence we have $C\left(p^{(n)}\right) \rightarrow 0$.

The following theorem is the main result in this section.

Theorem 2.1. Let $\left\{X_{n}, n \geq 0\right\}$ be a continuous-state homogeneous Markov chain with the transition probability density $p(x, y)$. The following propositions are equivalent:

(i) $\left\{X_{n}, n \geq 0\right\}$ is geometric strongly ergodic;

(ii) $\left\{X_{n}, n \geq 0\right\}$ is strongly ergodic;

(iii) $\left\{X_{n}, n \geq 0\right\}$ is weakly ergodic.

Remark. The results of this theorem are known (see [2], [7] and [10, p.384]). But here, we give a primary proof of this theorem.

Proof. (i) $\Rightarrow$ (ii) is obvious. Next we prove (ii) $\Rightarrow($ iii). Since

$$
\begin{aligned}
C\left(p^{(n)}\right) & =\frac{1}{2} \sup _{x_{1}, x_{2}} \int_{R}\left|p^{(n)}\left(x_{1}, y\right)-p^{(n)}\left(x_{2}, y\right)\right| d y \\
& \leq \frac{1}{2} \sup _{x_{1}} \int_{R}\left|p^{(n)}\left(x_{1}, y\right)-\pi(y)\right| d y+\frac{1}{2} \sup _{x_{2}} \int_{R}\left|p^{(n)}\left(x_{2}, y\right)-\pi(y)\right| d y,
\end{aligned}
$$

the weak ergodicity follows from the strong ergodicity of $\left\{X_{n}, n \geq 0\right\}$. 
Finally, we prove (iii) $\Rightarrow$ (i). Let $x_{0} \in R$, by Dobrushin inequality, for any $n, k$, we have

$$
\begin{aligned}
\int_{R}\left|p^{(n+k)}\left(x_{0}, y\right)-p^{(n+1)}\left(x_{0}, y\right)\right| d y & =\int_{R}\left|\int_{R}\left(p^{(k)}\left(x_{0}, z\right)-p\left(x_{0}, z\right)\right) p^{(n)}(z, y) d z\right| d y \\
& \leq \|\left(p^{(k)}\left(x_{0}, *\right)-p\left(x_{0}, *\right) \| C\left(p^{(n)}\right)\right. \\
& \leq 2 C\left(p^{(n)}\right) \rightarrow 0 \quad(n \rightarrow \infty) .
\end{aligned}
$$

Thus there exists a sequence such that

$$
\int_{R} p^{\left(n_{1}\right)}\left(x_{0}, y\right) d y+\sum_{k=1}^{\infty} \int_{R}\left|p^{\left(n_{k+1}\right)}\left(x_{0}, y\right)-p^{\left(n_{k}\right)}\left(x_{0}, y\right)\right| d y<\infty .
$$

It follows from (2.14) that

$$
p^{\left(n_{1}\right)}\left(x_{0}, y\right)+\sum_{k=1}^{\infty}\left(p^{\left(n_{k+1}\right)}\left(x_{0}, y\right)-p^{\left(n_{k}\right)}\left(x_{0}, y\right)\right) \text { converges a.e.. }
$$

On convergence points of $(2.15)$, let $\pi\left(x_{0}, y\right)=p^{\left(n_{1}\right)}\left(x_{0}, y\right)+\sum_{k=1}^{\infty}\left(p^{\left(n_{k+1}\right)}\left(x_{0}, y\right)-\right.$ $\left.p^{\left(n_{k}\right)}\left(x_{0}, y\right)\right)$, otherwise, let $\pi\left(x_{0}, y\right)=0$. It is easy to see that

$$
\lim _{k \rightarrow \infty} p^{\left(n_{k}\right)}\left(x_{0}, y\right)=\pi\left(x_{0}, y\right) \text { a.e.. }
$$

By (2.13), (2.16) and Fatou lemma, we have

$$
\int_{R}\left|p^{(n+1)}\left(x_{0}, y\right)-\pi\left(x_{0}, y\right)\right| d y \leq 2 C\left(p^{(n)}\right) .
$$

By $(2.17)$, we conclude that $\pi\left(x_{0}, y\right)$ is a transition probability density and $C(\pi)=0$, by Lemma $2.1, \pi\left(x_{0}, y\right)$ is a constant transition density $\left(\pi\left(x_{0}, y\right)=\pi(y)\right)$. Hence, for any $x$, we have

$$
\int_{R}\left|p^{(n+1)}(x, y)-\pi(y)\right| d y \leq 2 C\left(p^{(n)}\right) .
$$

Since $C\left(p^{(n)}\right) \rightarrow 0$, let $C\left(p^{\left(n_{0}\right)}\right)=r<1$ and $n=l n_{0}+k\left(k=0,1, \cdots, n_{0}-1\right)$, by (2.18), for any $x$, when $n \geq n_{0}$, we have

$$
\begin{aligned}
\int_{R}\left|p^{(n+1)}(x, y)-\pi(y)\right| d y \leq 2 C\left(p^{(n)}\right) & \leq 2 C\left(p^{\left(l n_{0}\right)}\right) \\
& \leq 2 r^{l}=2\left(r^{\frac{l}{n+1}}\right)^{n+1} \leq 2\left(r^{\frac{1}{2 n_{0}}}\right)^{n+1}=2 \delta^{n+1}
\end{aligned}
$$

where $0<\delta<1$. We obtain that $\left\{X_{n}, n \geq 0\right\}$ is geometric strongly ergodic. Thus we have completed the proof of this theorem. 


\section{STRONG LAW OF LARGE NUMBERS}

In this section, we will establish the strong law of large numbers for functions of $N+1$ variables of continuous-state nonhomogeneous Markov chains. Before proving the main result, we need to prove the following lemmas.

Lemma 3.1. Let $\left\{X_{n}, n \geq 0\right\}$ be a continuous-state nonhomogeneous Markov chain with the transition probability densities $\left\{p_{n}(x, y), n \geq 1\right\}$. Let $p(x, y)$ be another transition probability density. If

$$
\lim _{n \rightarrow \infty} \frac{1}{n} \sum_{k=1}^{n} \sup _{x}\left\|p_{k}(x, *)-p(x, *)\right\|=0,
$$

then for any positive integer numbers $m$ and $l$

$$
\lim _{n \rightarrow \infty} \frac{1}{n} \sum_{k=1}^{n} \sup _{x}\left\|p^{(m+k, m+k+l)}(x, *)-p^{(l)}(x, *)\right\|=0 .
$$

Proof. Now

$$
\begin{aligned}
& \sup _{x}\left\|p^{(m+k, m+k+2)}(x, *)-p^{(2)}(x, *)\right\| \\
= & \sup _{x} \int_{R}\left|p^{(m+k, m+k+2)}(x, y)-p^{(2)}(x, y)\right| d y \\
= & \sup _{x} \int_{R}\left|\int_{R} p_{m+k}(x, z) p_{m+k+1}(z, y) d z-\int_{R} p(x, z) p(z, y) d z\right| d y \\
\leq & \sup _{x} \int_{R}\left|\int_{R} p_{m+k}(x, z) p_{m+k+1}(z, y) d z-\int_{R} p_{m+k}(x, z) p(z, y) d z\right| d y \\
& +\sup _{x} \int_{R}\left|\int_{R}\left(p_{m+k}(x, z) p(z, y) d z-\int_{R} p(x, z) p(z, y)\right) d z\right| d y \\
\leq & \sup _{z}\left\|p_{m+k+1}(z, *)-p(z, *)\right\|+\sup _{z}\left\|p_{m+k}(z, *)-p(z, *)\right\| .
\end{aligned}
$$

It follows from (3.1) that for any $m$

$$
\lim _{n \rightarrow \infty} \frac{1}{n} \sum_{k=1}^{n} \sup _{x}\left\|p^{(m+k, m+k+2)}(x, *)-p^{(2)}(x, *)\right\|=0 .
$$

By induction, (3.2) holds.

Lemma 3.2. Let $\left\{X_{n}, \mathcal{F}_{n}, n \geq 0\right\}$ be a stochastic sequence on the probability space $(\Omega, \mathcal{F}, P)$, and let $\mathcal{F}_{-n}=\{\Omega, \phi\}, n \geq 1$. Let $\left\{\varphi_{n}, n \geq 1\right\}$ be a sequence of nonnegative, even functions defined on $R$ such that as $|x|$ increase

$$
\frac{\varphi_{n}(x)}{|x|} \uparrow, \quad \frac{\varphi_{n}(x)}{x^{2}} \downarrow .
$$


If

$$
\sum_{n=1}^{\infty} \frac{E\left[\varphi_{n}\left(X_{n}\right)\right]}{\varphi_{n}(n)}<\infty
$$

then for any $k \geq 1$

$$
\lim _{n \rightarrow \infty} \frac{1}{n} \sum_{m=1}^{n}\left\{X_{m}-E\left[X_{m+k} \mid \mathcal{F}_{m}\right]\right\}=0 \quad \text { a.e.. }
$$

Proof. By (3.5) and Corollary 1 of [5], for any $k \geq 1$, we have

$$
\lim _{n \rightarrow \infty} \frac{1}{n} \sum_{m=1}^{n}\left\{X_{m}-E\left[X_{m} \mid \mathcal{F}_{m-k}\right]\right\}=0 \quad \text { a.e.. }
$$

By using similar proof of Lemma 3 of [12]. By (3.5), we can obtain

$$
\lim _{n \rightarrow \infty} \frac{1}{n+k} E\left[X_{n+k} \mid \mathcal{F}_{n}\right]=0 \text { a.e., }
$$

(3.6) follows from (3.7) and (3.8).

The following theorem is the main result in this paper.

Theorem 3.1. Let $\left\{X_{n}, n \geq 0\right\}$ be a continuous-state nonhomogeneous Markov chain with the transition probability densities $\left\{p_{n}(x, y), n \geq 1\right\}$. Let $\left\{f_{n}, n \geq 1\right\}$ be a sequence of Borel measurable functions defined on $\mathcal{B}\left(R^{N+1}\right)$, and $\left\{\varphi_{n}, n \geq 1\right\}$ be the same as in Lemma 3.2. Let

$$
g_{n}(x)=\mathbf{E}\left[f_{n}\left(X_{n-N}, \cdots, X_{n}\right) \mid X_{n-N}=x\right],
$$

where $\sup _{x}\left|g_{n}(x)\right|<\infty$. Let $g(x)$ be another Borel function defined on $R$ such that $\sup _{x}|g(x)|<\infty$, and $p(x, y)$ be another transition probability density. Assume that $p(x, y)$ is strongly ergodic with probability density $\pi(y)$. If

$$
\begin{array}{r}
\sum_{n=1}^{\infty} \frac{E\left[\varphi_{n}\left(f_{n}\left(X_{n-N}, \cdots, X_{n}\right)\right)\right]}{\varphi_{n}(n)}<\infty, \\
\lim _{n \rightarrow \infty} \frac{1}{n} \sum_{m=1}^{n} \sup _{x}\left\|p_{m}(x, *)-p(x, *)\right\|=0,
\end{array}
$$

and

$$
\lim _{n \rightarrow \infty} \frac{1}{n} \sum_{m=1}^{n} \sup _{x}\left|g_{m}(x)-g(x)\right|=0
$$

then

$$
\lim _{n \rightarrow \infty} \frac{1}{n} \sum_{m=1}^{n} f_{m}\left(X_{m-N}, \cdots, X_{m}\right)=\int_{R} g(x) \pi(x) d x \quad \text { a.e. }
$$


Proof. Let $X_{-n}$ be a constant as $n \geq 1$, and let $\mathcal{F}_{n}=\sigma\left(X_{n}, X_{n-1}, \cdots\right)$. Then $\left\{f_{n}\left(X_{n-N}, \cdots, X_{n}\right), \mathcal{F}_{n}, n \geq 0\right\}$ is a stochastic sequence. By (3.10) and Lemma 3.2, we have for any $k \geq 1$

$$
\begin{array}{r}
\lim _{n \rightarrow \infty} \frac{1}{n} \sum_{m=1}^{n}\left\{f_{m}\left(X_{m-N}, \cdots, X_{m}\right)-E\left[f_{m+k+N}\left(X_{m+k}, \cdots, X_{m+k+N}\right) \mid \mathcal{F}_{m}\right]\right\} \\
=0 \text { a.e. }
\end{array}
$$

By Markov property and the properties of conditional expectation

$$
\begin{aligned}
& \frac{1}{n} \sum_{m=1}^{n} \mathbf{E}\left[f_{m+k+N}\left(X_{m+k}, \cdots, X_{m+k+N}\right) \mid \mathcal{F}_{m}\right] \\
& \quad=\frac{1}{n} \sum_{m=1}^{n} \mathbf{E}\left[\mathbf{E}\left[f_{m+k+N}\left(X_{m+k}, \cdots, X_{m+k+N}\right) \mid \mathcal{F}_{m+k}\right] \mid \mathcal{F}_{m}\right] \\
& \quad=\frac{1}{n} \sum_{m=1}^{n} \mathbf{E}\left[\mathbf{E}\left[f_{m+k+N}\left(X_{m+k}, \cdots, X_{m+k+N}\right) \mid X_{m+k}\right] \mid \mathcal{F}_{m}\right] \\
& \quad=\frac{1}{n} \sum_{m=1}^{n} \mathbf{E}\left[g_{m+k+N}\left(X_{m+k}\right) \mid X_{m}\right] \\
& \quad=\frac{1}{n} \sum_{m=1}^{n} \int_{R} g_{m+k+N}(x) p^{(m, m+k)}\left(X_{m}, x\right) d x .
\end{aligned}
$$

By (3.15), we have

$$
\begin{aligned}
&\left|\frac{1}{n} \sum_{m=1}^{n} E\left[f_{m+k+N}\left(X_{m+k}, \cdots, X_{m+k+N}\right) \mid \mathcal{F}_{m}\right]-\frac{1}{n} \sum_{m=1}^{n} \int_{R} g(x) p^{(k)}\left(X_{m}, x\right) d x\right| \\
&=\left|\frac{1}{n} \sum_{m=1}^{n} \int_{R} g_{m+k+N}(x) p^{(m, m+k)}\left(X_{m}, x\right) d x-\frac{1}{n} \sum_{m=1}^{n} \int_{R} g(x) p^{(k)}\left(X_{m}, x\right) d x\right| \\
& \leq \frac{1}{n} \sum_{m=1}^{n}\left|\int_{R} g_{m+k+N}(x) p^{(m, m+k)}\left(X_{m}, x\right) d x-\int_{R} g(x) p^{(m, m+k)}\left(X_{m}, x\right) d x\right| \\
& \quad+\frac{1}{n} \sum_{m=1}^{n}\left|\int_{R} g(x) p^{(m, m+k)}\left(X_{m}, x\right) d x-\int_{R} g(x) p^{(k)}\left(X_{m}, x\right) d x\right| \\
&=\frac{1}{n} \sum_{m=1}^{n}\left|\int_{R} p^{(m, m+k)}\left(X_{m}, x\right)\left(g_{m+k+N}(x)-g(x)\right) d x\right| \\
& \quad+\frac{1}{n} \sum_{m=1}^{n}\left|\int_{R} g(x)\left(p^{(m, m+k)}\left(X_{m}, x\right)-p^{(k)}\left(X_{m}, x\right)\right) d x\right| \\
& \leq \frac{1}{n} \sum_{m=1}^{n} \sup _{x}\left|g_{m+k+N}(x)-g(x)\right|
\end{aligned}
$$




$$
+\frac{1}{n} \sup _{x}|g(x)| \sum_{m=1}^{n} \sup _{x}\left\|p^{(m, m+k)}(x, *)-p^{(k)}(x, *)\right\| .
$$

By (3.12), the first term of right side of (3.16) converges to zero as $n \rightarrow \infty$. By (3.11) and Lemma 3.1, the second term of right side of (3.16) also converges to zero as $n \rightarrow \infty$. By (3.14) and (3.16), we have for any $k \geq 1$

$$
\lim _{n \rightarrow \infty} \frac{1}{n} \sum_{m=1}^{n}\left\{f_{m}\left(X_{m-N}, \cdots, X_{m}\right)-\int_{R} g(x) p^{(k)}\left(X_{m}, x\right) d x\right\}=0 \quad \text { a.e.. }
$$

Now

$$
\begin{aligned}
& \left|\frac{1}{n} \sum_{m=1}^{n} \int_{R} g(x) p^{(k)}\left(X_{m}, x\right) d x-\int_{R} g(x) \pi(x) d x\right| \\
& \quad \leq \frac{1}{n} \sum_{m=1}^{n} \int_{R}\left|g(x)\left(p^{(k)}\left(X_{m}, x\right)-\pi(x)\right)\right| d x \\
& \quad \leq \frac{1}{n} \sum_{m=1}^{n} \sup _{x}|g(x)| \int_{R} \mid\left(p^{(k)}\left(X_{m}, x\right)-\pi(x) \mid d x\right. \\
& \quad \leq \sup _{x}|g(x)| \sup _{y} \int_{R}\left|p^{(k)}(y, x)-\pi(x)\right| d x .
\end{aligned}
$$

Since $p(y, x)$ is strongly ergodic with probability density $\pi(x)$, the right side of (3.18) is small provided $k$ is large, equation (3.13) follows from (3.17) and (3.18) directly.

Corollary 3.1. Let $\left\{X_{n}, n \geq 0\right\}$ be a continuous-state nonhomogeneous Markov chain with the transition probability densities $\left\{p_{n}(x, y), n \geq 1\right\}$. Let $\left\{f_{n}, n \geq 1\right\}$ be a sequence of Borel measurable functions defined on $\mathcal{B}\left(R^{2}\right)$, and $\left\{\varphi_{n}, n \geq 1\right\}$ be the same as in Lemma 3.2. Let

$$
g_{n}(x)=E\left[f_{n}\left(X_{n-1}, X_{n}\right) \mid X_{n-1}=x\right]
$$

where $\sup _{x}\left|g_{n}(x)\right|<\infty$. Let $g(x)$ be another Borel function defined on $R$ such that $\sup _{x}|g(x)|<\infty$, and $p(x, y)$ be another transition probability density. Assume that $p(x, y)$ is strongly ergodic with probability density $\pi(y)$. If (3.11) and (3.12) hold, and

$$
\sum_{n=1}^{\infty} \frac{E\left[\varphi_{n}\left(f_{n}\left(X_{n-1}, X_{n}\right)\right)\right]}{\varphi_{n}(n)}<\infty
$$

then

$$
\lim _{n \rightarrow \infty} \frac{1}{n} \sum_{m=1}^{n} f_{m}\left(X_{m-1}, X_{m}\right)=\int_{R} g(x) \pi(x) d x \quad \text { a.e.. }
$$

Proof. Letting $N=1$ in Theorem 3.1, this corollary follows. 
Corollary 3.2. Let $\left\{X_{n}, n \geq 0\right\}$ be a continuous-state nonhomogeneous Markov chain with the transition probability densities $\left\{p_{n}(x, y), n \geq 1\right\}$. Let $p(x, y)$ be another transition probability density. Assume that $p(x, y)$ is strongly ergodic with probability density $\pi(y)$. Let $S_{n}^{B}(\omega)(B \in \mathcal{B}(R))$ be the times of visiting $B$ of $X_{0}, X_{1}, \cdots, X_{n-1}$, that is

$$
S_{n}^{B}(\omega)=\sum_{m=0}^{n-1} I_{\left\{X_{m \in B}\right\}} .
$$

If (3.11) holds, then

$$
\lim _{n \rightarrow \infty} \frac{S_{n}^{B}(\omega)}{n}=\int_{B} \pi(y) d y \quad \text { a.e.. }
$$

Proof. Letting $f_{n}(x, y)=I_{B}(y)$ in Corollary 3.1, and $\varphi_{n}=x^{2}$, obviously (3.10) holds, and

$$
g_{n}(x)=\int_{R} I_{B}(y) p_{n}(x, y) d y=\int_{B} p_{n}(x, y) d y
$$

Let

$$
g(x)=\int_{B} p(x, y) d y
$$

by $(3.11)$, we have $(3.12)$ holds. Since $p(x, y)$ is strongly ergodic with probability density $\pi(y)$, then $\pi(y)$ is the stationary distribution determined by $p$, we have

$$
\begin{aligned}
\int_{R} g(x) \pi(x) d x=\int_{R}\left(\int_{B} p(x, y) d y\right) & \pi(x) d x \\
= & \int_{B}\left(\int_{R} p(x, y) \pi(x) d x\right) d y=\int_{B} \pi(y) d y .
\end{aligned}
$$

By (3.23) and Corollary 3.1, we have

$$
\begin{aligned}
& \lim _{n \rightarrow \infty} \frac{1}{n} \sum_{m=1}^{n} f_{m}\left(X_{m-1},\right.\left.X_{m}\right)=\lim _{n \rightarrow \infty} \frac{1}{n} \sum_{m=1}^{n} I_{\left\{X_{m} \in B\right\}} \\
&=\lim _{n \rightarrow \infty} \frac{1}{n} S_{n}^{B}(\omega)=\int_{R} g(x) \pi(x) d x=\int_{B} \pi(y) d y \text { a.e.. }
\end{aligned}
$$

Thus (3.22) holds.

\section{ACKNOWLEDGMENTS}

This work was supported by the National Natural Science Fundation of China 11571142. 


\section{REFERENCES}

[1] Z. Dietz and S. Sethuraman, Large deviations for a class of nonhomogeneous Markov chains, Ann. Appl. Probab., 15 (2005), 421-486.

[2] R.L. Dobrushin, Central limit theorems for non-stationary Markov chains I, II, Theor. Probab. Appl., 1 (1956), 65-80 and 329-383.

[3] G.X. Liu and W. Liu, On the strong law of large numbers for functionals of countable nonhomogeneous Markov chains, Stochastic Process. Appl., 50 (1994), 375-391.

[4] W. Liu and G.X. Liu, A class of strong laws for functionals of countable nonhomogeneous Markov chains, Statist. Probab. Lett., 22 (1995), 87-96.

[5] W. Liu and W.G. Yang, A class of strong limit theorems for the sequences of arbitrary random variables, Statist. Probab. Lett., 64 (2003), 121-131.

[6] W. Liu and W.G. Yang, An extension of Shannon-McMillan theorem and some limit properties for nonhomogeneous Markov chains, Stochastic Process. Appl., 61 (1996), 129-145.

[7] F. Mukhamedov, Ergodic properties of nonhomogeneous Markov chains defined on ordered banach spaces with a base, Acta Math. Hungar., 147 (2015), 294-323.

[8] R.W. Madsen and D.L. Isaacson, Strongly ergodic behavior for non-stationary Markov processes, Ann. Probab., 1 (1973), 329-335.

[9] R.W. Madsen, A note on some ergodic theorems of A, Paz. Ann. Math. Statist, 42 (1971), 405-408.

[10] S.P. Meyn and R.L. Tweedie, Markov Chains and Stochastic Stability, SpringerVerlag, New York, 1993.

[11] W.G. Yang, Convergence in the Cesàro sense and strong law of large numbers for nonhomogeneous Markov chains, Linear Algebra Appl., 354 (2002), 275-288.

[12] W.G. Yang, Strong law of large numbers for countable nonhomogeneous Markov chains, Linear Algebra Appl., 430 (2009), 3008-3018. 
